\title{
Aisha Email System
}

\author{
Aisha Hamad Abobaker ${ }^{1}$, Surya Prakash Mishra ${ }^{2}$ \\ ${ }^{1}$ M.Sc. in Computer Science in Department of Computer Science and Information Technology in SHIATS, Allahabad, India. \\ ${ }^{2}$ Asst. Prof. in the Department of Computer Science and Information Technology in SHIATS, Allahabad, India.
}

\begin{abstract}
The Paper Entitle "Aisha Email System" deals with identifying the clients to send and receive mail with the same login. This utility will allow multiple clients to login under the same login name and still have personalized mail information, enabling them to send and receive mails. Each user willing to avail the services offered by the mail server application should exist as a user before he can send or receive mails. This is made possible by prompting each user to enter his user-id and password before he can send or view his mails. This Paper has Inbox, compose and address list. E-mail is one of the most common and reliable methods of communication for both personal and business purposes. It also plays an important role in each and every Web site. This role will be in the type of automated e-mails from the server after posting information from a form.

The Paper has been planned to be having the view of distributed architecture, with centralized storage of the database. The application for the storage of the data has been planned. Using the constructs of MS-SQL Server and all the user interfaces have been designed using the ASP.Net technologies. The database connectivity is planned using the "SQL Connection" methodology. The standards of security and data protective mechanism have been given a big choice for proper usage. The application takes care of different modules and their associated reports, which are produced as per the applicable strategies and standards that are put forwarded by the administrative staff.

The entire Paper has been developed keeping in view of the distributed client server computing technology, in mind. The specification has been normalized up to $3 \mathrm{NF}$ to eliminate all the anomalies that may arise due to the database transaction that are executed by the general users and the organizational administration. The user interfaces are browser specific to give distributed accessibility for the overall system. The internal database has been selected as MS-SQL server 200.The basic constructs of table spaces, clusters and indexes have been exploited to provide higher consistency and reliability for the data storage.

The MS-SQL server 200 was a choice as it provides the constructs of high-level reliability and security. The total front end was dominated using the ASP.Net technologies. At all proper levels high care was taken to check that the system manages the data consistency with proper business rules or validations. The database connectivity was planned using the latest "SQL Connection" technology provided by Microsoft Corporation. The authentication and authorization was crosschecked at all the relevant stages. The user level accessibility has been restricted into two zones namely.
\end{abstract}

\section{INTRODUCTION}

This Paper is about developing a web based mail client connecting to windows Server running a Mail Server. This system has the following main functionality

1. Receiving/Sending/organizing mails.

2. Sending mail using send mail.

3. Performing Admin functions like managing new user, resetting passwords etc.

Software Solutions is an IT solution provider for a dynamic environment where business and technology strategies converge. Their approach focuses on new ways of business combining IT innovation and adoption while also leveraging an organization's current IT assets. Their work with large global corporations and new products or services and to implement prudent business and technology strategies in today's environment.

- Software Development Services

- Engineering Services

- Systems Integration

- Customer Relationship Management

- $\quad$ Product Development

- Electronic Commerce

- Consulting

- $\quad$ IT Outsourcing

We apply technology with innovation and responsibility to achieve two broad objectives:

- Effectively address the business issues our customers face today. 
- Generate new opportunities that will help them stay ahead in the future.

- A strategy where we architect, integrate and manage technology services and solutions - we call it AIM for success.

- A robust offshore development methodology and reduced demand on customer resources.

- A focus on the use of reusable frameworks to provide cost and times benefits.

They combine the best people, processes and technology to achieve excellent results - consistency. We offer customers the advantages of:

\section{Related Work:}

They understand the importance of timing, of getting there before the competition. A rich portfolio of reusable, modular frameworks helps jump-start projects. Tried and tested methodology ensures that we follow a predictable, low - risk path to achieve results. Our track record is testimony to complex projects delivered within and evens before schedule.

Our teams combine cutting edge technology skills with rich domain expertise. What's equally important - they share a strong customer orientation that means they actually start by listening to the customer. They're focused on coming up with solutions that serve customer requirements today and anticipate future needs.

They offer customers the advantage of being able to Architect, integrate and manage technology services. This means that they can rely on one, fully accountable source instead of trying to integrate disparate multi vendor solutions., providing it's services to companies which are in the field of production, quality control etc With their rich expertise and experience and information technology they are in best position to provide software solutions to distinct business requirements.

\section{Proposed Work:}

This Paper is developed to create a Mail Server. A Mail server is an application which used to send and receive mails. This type of application needs to manage mails send by registered users. A message has to be composed in compose box. This message is stored in inbox directory of receiver. This file should be combination of the sender's user-id and sub. The file transmitted is moved from sender compose to receiver inbox. The recipient can view the message by selecting the appropriate file no. This is also provision for the replying and deleting the message. This utility also enables users, working under different login names to communicate with each other. We can send files through attachments. In this we attach the files to the mail by browsing option. From desktop or any drive we can select files and we will attach them to the mail that we want to send presently and then we send them to the receiver.

- Capability to create user Email Accounts by an Administrator or by End users after registering themselves

- Administrator functionality to Delete User Accounts, Change passwords

- Capability for End users to login into the system using a browser

- Capability for logged in users to send/receive/forward/reply/delete mails

- Invalidate user login on inactive for more than $10 \mathrm{mts}$

- Address book capability

- Mark mails as Junk

- Apply Label to Mail

- Organize mails in Logical Folders

\section{Problem In EXISTING System}

- Cannot Upload and Download the latest updates.

- No use of Web Services and Remoting.

- Risk of mismanagement and of data when the Paper is under development.

- Less Security.

- No proper coordination between different Applications and Users.

- Fewer Users - Friendly.

- Manual system need man power a lot.

- Communication between Patient and administration is a tuff job.

- Difficult to maintain each and patient information in form of files.

\section{Solution Of These Problems}

The development of the new system contains the following activities, which try to automate the entire process keeping in view of the database integration approach.

User friendliness is provided in the application with various controls. 
The system makes the overall Paper management much easier and flexible.

Readily upload the latest updates, allows user to download the alerts by clicking the URL.

There is no risk of data mismanagement at any level while the Paper development is under process.

It provides high level of security with different level of authentication.

\section{Benefits}

The Paper is identified by the merits of the system offered to the user. The merits of this Paper are as follows: -

- It's a web-enabled project.

- This Paper offers user to enter the data through simple and interactive forms. This is very helpful for the client to enter the desired information through so much simplicity.

- The user is mainly more concerned about the validity of the data, whatever he is entering. There are checks on every stages of any new creation, data entry or updation so that the user cannot enter the invalid data, which can create problems at later date.

- Sometimes the user finds in the later stages of using Paper that he needs to update some of the information that he entered earlier. There are options for him by which he can update the records. Moreover there is restriction for his that he cannot change the primary data field. This keeps the validity of the data to longer extent.

- User is provided the option of monitoring the records he entered earlier. He can see the desired records with the variety of options provided by him.

- From every part of the Paper the user is provided with the links through framing so that he can go from one option of the Paper to other as per the requirement. This is bound to be simple and very friendly as per the user is concerned. That is, we can sat that the Paper is user friendly which is one of the primary concerns of any good project.

- Data storage and retrieval will become faster and easier to maintain because data is stored in a systematic manner and in a single database.

- Decision making process would be greatly enhanced because of faster processing of information since data collection from information available on computer takes much less time then manual system.

- Easier and faster data transfer through latest technology associated with the computer and communication.

- Through these features it will increase the efficiency, accuracy and transparency,

\section{CONCLUSION}

It has been a great pleasure for me to work on this exciting and challenging project. This Paper proved good for me as it provided practical knowledge of not only programming in ASP.NET and VB.NET web based application and no some extent Windows Application and SQL Server, but also about all handling procedure related with "PAPER NAME". It also provides knowledge about the latest technology used in developing web enabled application and client server technology that will be great demand in future. This will provide better opportunities and guidance in future in developing projects independently.

\section{REFERENCES}

[1] Albert Levi and Mahmut Özcan(2012)Practical and Secure E-Mail System (PractiSES) BBXX0937X37JJ37

[2] J.S. Allen(2011) Mailing System 979-0; 979-1

[3] M.S. Sanga(2012) Post by Email ISB/0001837X373/09

AUTHOR'S PROFILE

AISHA HAMAD ABOBAKER: Received her Bachelor of Computer from Faculty of science Sebha in LIBYA 2007, M.Sc. in Computer Science in Department of Computer Science and Information Technology in SHIATS, Allahabad, India.

SURYA PRAKASH MISHRA: Master of Computer Application from UP. Tech. University, Lucknow - in 2005, Asst. Prof. in the Department of Computer Science and Information Technology in SHIATS, Allahabad, India. 